\title{
ARTICLES
}

\section{Penelopegate : fake news et retravail d'ethos en ligne lors de la campagne présidentielle de 2017}

\author{
Penelopegate: fake news and online fake work \\ over the 2017 French presidential campaign
}

\author{
Fabienne H. Baider, Maria Constantinou \\ Université de Chypre \\ helenafab@yahoo.fr,mariacon_2004@yahoo.com
}

\begin{abstract}
The French politician François Fillon, for a long time considered the frontrunner in the French presidential campaign, saw his strong position crumble to the third in most of the polls, after the circulation of rumors against him and his family also known as Penelopegate. Such rumors were at the heart of the 2017 French and American presidential campaigns. In fact, the terms post truth, fake news and alternative facts could be seen as symbols of the current information crisis, i.e. the mistrust felt by many readers regarding the media. The present study sets out to investigate the argumentation used to deconstruct and reconstruct François Fillon's ethos after dissemination of such rumors on social media. Drawing on the theoretical framework developed by Amossy (2014 et al.) and Maingueneau (2004 et al.) on the analysis of self-presentation and in particular on the concepts of (re)branding and scenography, we analyze the discursive strategies deployed by discussants in face management of the French politician's image. Our data include two different genres (tweets and posts on newspaper forums) and our findings corroborate Amossy's research (2014) on strategies used in such face work management as denial of responsibility (sympathy), victimization (empathy) or face threats against the opponents (anger). We have also observed the use of a catastrophic scenario (fear), which was not present in Amossy's data.
\end{abstract}

Keywords: ethos, fake news, François Fillon, emotions, rebranding, social media. 


\title{
INTRODUCTION
}

\author{
Fillon en mode « fait alternatif » à la Trump... \\ Mentez! Mentez ! Il en restera toujours quelque chose !
}

Les tweets mis en exergue illustrent l'importance des (faux) bruits colportés le plus souvent sur les réseaux sociaux dans la perception de l'ethos des hommes politiques. Les rumeurs ont été de fait au cœur des campagnes présidentielles de 2017, française et américaine et les termes de post-vérité (post truth), fake news (fausses nouvelles), 'alternative facts' (faits alternatifs) pourraient être considérés comme des symboles de la défiance ressentie par de nombreux lecteurs envers les médias : tout le monde ne serait-il pas dans le mensonge ou dans une réalité/vérité qui évoluerait selon l'identité du locuteur ou de la locutrice?

L'objectif de notre article est d'observer et d'analyser comment l'ethos de l'homme politique François Fillon s'est construit, déconstruit et reconstruit au fil des commentaires relatifs à la série de révélations et à la circulation des rumeurs sur les réseaux sociaux et sur les forums de journaux.

Fillon était le grand favori des élections présidentielles 2017 jusqu'au 24 janvier 2017, date où des emplois fictifs de Pénélope Fillon, sa femme, ont été révélés par Le Canard enchaîné ; cette nouvelle fut suivie d'autres bruits et accusations fondées ou mensongères et, entre autres, la rumeur du suicide de sa femme ; il termina $3^{\mathrm{e}}$ lors du premier tour des élections en mai 2017. Afin d'analyser les stratégies discursives choisies par ses partisans pour contrer les différentes révélations, nous travaillons avec les notions suivantes développées dans de nombreux travaux de Ruth Amossy (2014, inter alia) et de Dominique Maingueneau (2004, inter alia) : le retravail de l'ethos, l'ethos dit/l'ethos montré et la scénographie (Maingueneau, 2004). L'étude de R. Amossy (2014) sur le travail d'ethos de Ségolène Royal lors de son échec aux présidentielles de 2007 sera notre base pour analyser les stratégies des Fillonistes (adoptées aussi par F. Fillon), celles-ci étant sensiblement identiques à celles adoptées par la femme politique socialiste. Pour ce faire, dans la première partie, nous situons la « scénographie » lors de la rumeur $\mathrm{du}$ suicide, et introduisons cette notion de retravail d'ethos. Sa construction au fil des mots est analysée dans deux types de données en ligne: les commentaires d'internautes laissés sur Twitter (deuxième section) et les commentaires postés sur des forums des articles de journaux français, articles ayant été relayés sur Twitter (troisième section).

\footnotetext{
${ }^{1}$ http://www.liberation.fr/direct/element/_59182/ ...pic.twitter.com/w5YqkdpSSK
} 


\section{SCÉNOGRAPHIE, BRANDING, TRAVAIL D’ETHOS}

Cette section pose les jalons théoriques qui sous-tendent notre étude, notamment les paramètres imposés par le contexte discursif du discours politique et du discours sur les politiques.

\section{1. (RE)PERSUADER SON PUBLIC : QUELLE SCÉNOGRAPHIE POUR LES FILLONISTES ?}

Le cadre institutionnel qu'est la campagne présidentielle française exige des candidats de se conformer à certaines normes de dire, de faire et de paraître ${ }^{2}$. Tout Twitter ou commentaire d'internaute s'inscrit dans la vaste formation discursive que composent les réseaux intertextuels et transnationaux, ainsi une comparaison favorite des internautes est le parallèle entre le président américain Trump et l'homme politique français. Il s'agit donc de prendre compte lors de notre analyse la « scénographie » des dires (Maingueneau, 2004, p. 160), c'est-à-dire les variables qui gèrent l'énonciation d'un discours singulier.

Ces variables incluent plusieurs dimensions du contexte de l'énonciation. Ainsi l'énonciation des commentateurs se doit-elle d'abord de respecter des modèles culturels $^{3}$. Elle est aussi intertextuelle, faisant référence à l'ensemble des dires $d e$ et sur des personnes politiques étudiées; nous tenons compte des temps forts depuis le début des primaires des différents partis politiques lors de la campagne des élections présidentielles françaises de 2017. De plus, des contraintes matérielles propres à l'énonciation sur des plateformes Twitter et des forums formatent un genre d'énonciation : il est notoire que le nombre de caractères sur Twitter est limité, et les participants doivent ainsi adapter leurs interventions aux contraintes de la plateforme. Enfin, le discours énoncé présuppose aussi une « scénographie » spécifique pour pouvoir être énoncé « et qu'en retour il doit valider à travers son énonciation » (Mainguenau, 2004, p. 192).

Compte tenu de ces paramètres, les détracteurs et les défenseurs de F. Fillon vont jouer sur différents présupposés et topoï argumentatifs pour valider leur positionnement ou invalider celui de l'autre. Ces échanges sont importants politiquement car l'entité de Fillon renvoie aussi à l'image symbolique d'un parti, et donc un ensemble d'électeurs potentiels qui doivent soit se reconnaître dans les dires, soit être en accord avec le faire :

\footnotetext{
${ }^{2}$ Ainsi les multiples remarques sur les choix de conduite et de vêtements du candidat anticapitaliste Poutou.

${ }^{3}$ Ainsi si l'ironie est particulièrement mordante chez les internautes français dans nos données, elle peut être moindre dans d'autres cultures internautes. L'agressivité est aussi semble-t-il plus le fait de commentateurs polonais que britanniques (Lewandowska-Tomaszczyk, 2017).
} 
Le discours politique n'est pas seulement à concevoir comme une prise en compte des aspirations des électeurs, il est aussi performatif au sens où il constitue une certaine réalité dans laquelle les citoyens peuvent se reconnaître même s'ils n'étaient pas initialement destinés à l'être ${ }^{4}$. (Longhi, 2016b)

L'autorité et la légitimité de la personne politique ne sont donc pas uniquement conférées par le pouvoir institutionnel, mais aussi par cette «agentivité rhétorique » de ces partisans ou de ces détracteurs (Amossy, 2014, p. 22) : 1'entreprise de persuasion des commentateurs est donc orientée par leur choix de scénographie et le topos qui la surplombe (Fillon est un menteur effronté vs Fillon est la victime d'un complot). L'ethos relève aussi de cette scénographie (Maingueneau, 2004, pp. 207-212) car il est le «garant» du discours : il convoque les valeurs qui doivent susciter l'adhésion de l'auditoire ciblé.

\subsection{BRANDING POLITIQUE ET ETHOS PRÉDISCURSIF}

Le mot ethos peut être défini et travaillé de manière diverse dans la mesure où il est l'objet d'études en rhétorique, pragmatique, analyse de discours ou sociologie (Perelman et Olbrechts-Tyteca, 1992 ; Amossy, 1999, 2010, 2014 ; Chauvin, 2002). Dans cet article, nous adoptons la définition proposée par Ruth Amossy, c'est-à-dire « l'image que l'orateur construit de sa propre personne pour assurer sa crédibilité » (2014, p. 13), tout en retenant que cette image est avant tout un ensemble de valeurs attendues par le public cible comme précisé plus haut. Cet ensemble de valeurs construit aussi de manière dialectique la représentation de Fillon dans l'interlocution (Adam, 1999 ; Chauvin, 2002 ; Barthes, 1962 ; Eggs, 1999 ; Woerther, 2007; Amossy, 2014, inter alia $)^{5}$. Une telle présentation de soi en politique a aussi été dénommée branding (Amossy, 2014, p. 20) : « on parle désormais de branding en matière de promotion de personnes politiques en particulier en période d'élections ». Dans cette promotion de la personne, l'argumentation a une place de choix car son efficacité est liée à l'efficacité de la parole, elle-même pouvant être conditionnée par «l'autorité de l'orateur » (Amossy, 1999, p. 127).

Nous observerons les tentatives de réparation de ce branding face à la rumeur dans notre troisième partie, soit le face work ou face management de Goffman (1973; Benoit, 1995) qu'exercent les militants pour réajuster l'ethos de Fillon et pour reconstruire sa présidentiabilité. Ainsi le Bart (2009) explique qu'un ou une présidentiable, selon la conception du pouvoir en France, doit construire une identité stable, une grande force psychologique et une maîtrise de soi. L'étude de fréquences lexicales dans la presse française ont aussi proposé un profil sémantique des tenants

\footnotetext{
${ }^{4} \mathrm{http}$ :/theconversation.com/performativite-et-performance-du-discours-politique-de-francois-fillon-70425

${ }^{5}$ Comme en témoigne l'attention des personnes politiques accordée aux sondages d'opinion.
} 
de la « présidentiabilité » des candidats, notamment la crédibilité, la légitimité, et la compétence (Baider, 2015) ${ }^{6}$. Dans le cas des personnes politiques en difficulté, un face work, pour démontrer ces qualités se pratique à tous les instants par la personne politique, et ses militants, « une entreprise délibérée et systématique de réparation de son image » (Amossy, 2014, p. 24 ; nos italiques) lors d'attaques et donc un réajustement des paramètres de crédibilité, de légitimité et de compétence.

De fait, les quelques études qui ont travaillé sur l'ethos de François Fillon (Longhi, 2016a ; Lebaron, 2017) ont montré que le lexique de valeurs (valeur, vérité) soutenant l'argument de crédibilité et le besoin de changement important (redresser, changer, radical) appuyant celui de compétence caractérisaient les discours de l'homme politique, mais aussi ses actions. En effet, alors que Marine Le Pen a refusé de se rendre à une convocation des juges d'instruction quant à des emplois fictifs au parlement européen ${ }^{7}$, Fillon s'y est rendu, conforme à l'ethos construit selon ses dires, ceux des militants et ceux de la presse. De fait, Fillon avait assis sa crédibilité notamment sur la figure d'un "Monsieur Propre » de la politique jusque fin janvier $2017^{8}$ : «L'ethos de François Fillon (...) était celui d'un chrétien discret, travailleur, fondamentalement honnête, intègre avec l'argent public et 'bon père de famille', droit et conséquent » (Lebaron, 2017) ${ }^{9}$.

Ce capital symbolique de chevalier blanc de la politique (fondamentalement honnête, intègre avec l'argent public et " bon père de famille », droit et conséquent) lui a permis de gagner largement face à ses adversaires à la primaire, notamment contre N. Sarkozy. Selon les pronostics mettant tous Marine Le Pen présente au $2^{\mathrm{e}}$ tour, sans le «Pénélopegate », il était sûr d’être élu.

\subsection{ACCUSATION, RUMEURS ET DE-CONSTRUCTION D'ETHOS}

Cet ethos savamment construit a été mis en danger lors de la révélation des contrats soupçonnés d'être fictifs de travail pour sa femme et ses enfants :

Dévastateur. (...) Il risque surtout de perdre une partie de l'électorat populaire des classes moyennes et modestes qui auraient voté pour lui, mais qui sont choquées par, pensent-elles, sa duplicité. (Faye, 2017)

De ce fait, une fausse nouvelle, l'annonce du suicide de sa femme, a pris plus d'ampleur. En effet, le 5 mars 2017 lors de son passage au 20-Heures de France 2,

\footnotetext{
${ }^{6}$ Amossy (2014, p. 22) associe autorité tour à tour à crédibilité et à légitimité.

${ }^{7} \mathrm{http}: / /$ www.huffingtonpost.fr/2017/07/01/mise-en-examen-dans-laffaire-des-emplois-fictifs-au-par lement-e_a_23011156/. Les membres du parlement bénéficient d'une immunité parlementaire.

${ }^{8} \mathrm{http} / / / \mathrm{www}$. lexpress.fr/actualite/politique/elections/fillon-monsieur-propre-et-irreprochable-des-su rnoms-qui-n-ont-plus-de-chien_1875466.html

${ }^{9} \mathrm{http}: / /$ www.medelu.org/Une-affaire-d-ethos-le-cas-Fillon
} 
François Fillon affirme être la victime de calomnies médiatiques et tout en exprimant son désarroi, accuse les chaînes de télévision d'avoir évoqué le suicide de son épouse. La déclaration du suicide de sa femme vient juste après la maladresse du candidat déclarant « je ne suis pas autiste » lors de la même émission ${ }^{10}$ pour justifier qu'il était à l'écoute de son propre camp. À cela s'ajoute le chiffre exagéré des 200000 personnes qui selon les équipes du candidat auraient été présentes au rassemblement du 5 mars au Trocadéro. Tant la bévue « je ne suis pas autiste » que le chiffre exagéré pour le Trocadéro ont suscité des réactions sur les réseaux sociaux et ont fait l'objet de nombreux articles journalistiques. La fausse annonce du suicide de sa femme a provoqué également des réactions sur les réseaux sociaux où les internautes et les journalistes ont toute de suite commencé à chercher la source d'une telle information sur Internet. La seule source évoquant le suicide de sa femme identifiée jusqu'à lors sur Internet était un tweet du $1^{\text {er }}$ mars venant de Madeleine de Jessey, l'une de ses proches, qui accusait à tort les journalistes d'avoir annoncé le suicide de la femme de Fillon. À côté de cette supposée annonce, le même homme politique a fait référence, pendant son discours pour la journée de la femme le 8 mars, à un jeu sordide impliquant des lames de rasoir et de jeunes filles en jupes courtes. S'agissant de « deux affaires dont personne n'a trouvé trace, à l'exception de François Fillon et ses proches $"{ }^{11}$, les médias et les réseaux sociaux accusent le candidat d'être devenu adepte de fake news promues par l'administration Trump pour manipuler l'opinion publique, afin d'appuyer sa candidature à l'élection présidentielle.

Dans ce contexte, le plus important pour les soutiens de l'homme politique était donc de retrouver l'adéquation entre la personne, son verbe et les circonstances (Amossy, 2002), adéquation que les rumeurs avaient mise en doute ou détruite; il ne s'agissait plus de jouer au " chevalier blanc ", mais de retrouver un minimum d'honnêteté et de crédibilité, indispensables pour un présidentiable. Les tentatives de réorientation ou même de transformation de l'image détériorée se feront à travers le pouvoir des mots des supporters sur les réseaux sociaux qui s'ajoute à celui institutionnel du chef de parti (Amossy, 2011, p. 22) ; de fait, les stratégies mises en place dans les discours d'une part et sur les réseaux sociaux d'autre part se sont fait écho.

Sachant que les réseaux sociaux, tels que Facebook et Twitter, sont des outils de propagation rapide, l'enjeu était de taille ; il fallait vite mettre en place des stratégies discursives pouvant effectivement réajuster tant soit peu l'adéquation entre 'ethos dit' (les teneurs du discours de Fillon) et 'ethos montré' (les révélations médiatiques).

${ }^{10} \mathrm{http} / / /$ www.lefigaro.fr/elections/presidentielles/2017/03/06/35003-20170306ARTFIG00089-enaffirmant-je-ne-suis-pas-autiste-fillon-suscite-le-polemique.php. La citation est la suivante : « je ne suis pas autiste, je vois bien les difficultés, j'entends bien les critiques ».

${ }^{11} \mathrm{http} / / / \mathrm{www}$.europe1.fr/politique/jeu-sordide-et-suicide-de-penelope-francois-fillon-se-defend-dediffuser-des-fake-news-3001376 
Il s'agissait de 'sauver la face' et de trouver un compromis entre les désirs de son électorat de droite, les besoins du cadre institutionnel (le parti Les Républicains), le pré-ethos discuté précédemment qui lui a permis de gagner à la primaire et l'ethos endommagé.

Pour étudier les stratégies mises en place, nous avons choisi deux genres discursifs : l'un comprend des commentaires postés par des internautes sur des forums de journaux et l'autre des tweets. L'articulation de ces deux corpus différents se justifie par leur complémentarité : alors que les tweets consistent en des messages plus courts en raison des contraintes d'espace imposées par Twitter, les forums donnent la possibilité aux intervenants d'argumenter et exprimer leurs sentiments.

\section{TWITTER OU LA VAGUE ANTI-FILLON}

Nous avons d'abord procédé à une étude basée sur des échanges twittés et notamment les échanges les plus populaires.

\subsection{UNE NÉGATIVITÉ GÉNÉRALISÉE}

En effet, la plateforme Twitter est de plus en plus utilisée pour les échanges publics et les personnes et campagnes politiques se font fort d'occuper le terrain sur ce réseau social ${ }^{12}$. Si nous observons un manque d'argumentation dans les échanges, notre corpus atteste aussi de l'importance de la plateforme pour la circularité des (fausses) nouvelles si ce n'est des arguments.

Suivant la méthodologie de Longhi (2016a) pour l'analyse quantitative, nous nous sommes limitées aux tweets les plus partagés pour cette étude. La récolte du corpus de tweets a été réalisée à l'aide des mots clés définissant l'événement discursif en question, comme Penelope Fillon suicide ou Penelope Fillon fake news afin d'étudier les réactions des internautes autour de l'ethos de Fillon après la circulation de la fausse information du suicide de sa femme. Les deux corpus ont été récoltés vers fin mai (24-27 mai 2017).

Il est à noter que parmi les 1279 tweets, seulement 195 ont été retweetés plus de 8 fois. Ayant sélectionné les tweets les plus partagés, nous avons aussi pris en compte les tweets les plus aimés. Nous ferons l'hypothèse qu'ils représentent les croyances les plus communes concernant le candidat à la présidentielle (Longhi, 2016a), exprimées le plus souvent de manière ludique, l'humour étant la dimension la plus

\footnotetext{
${ }^{12} \mathrm{http} / / /$ www.la-croix.com/France/Politique/Comment-Twitter-change-communication-politique2016-07-13-1200775629
} 
prégnante de notre corpus. En particulier, une ironie mordante met en avant le manque d'équation entre la représentation préexistante de Fillon avant les révélations (son pré-ethos aux événements) et celui que révèlent ses actions.

\subsection{TWEET LE PLUS PARTAGÉ, LE PLUS FAVORISÉ}

Dans notre étude, les tweets les plus populaires construisent une corrélation entre fond et forme sémantiques du nom propre Fillon, au moyen de « la récurrence, de la stabilisation et de la circulation » (Longhi, 2016a). Les moyens mis en œuvre par les twittants servent soit à montrer l'incohérence du nom propre Fillon avec la fonction de président, soit au contraire à rétablir une harmonie entre ethos dit et ethos montré, de façon ironique ; ce jeu se fait par le retravail du déjà dit et par « la modulation de ces images verbales préexistantes » (Amossy, 2014, p. 24).

De fait, le classement des tweets selon leur axiologie montre qu'à $70 \%$ ils sont négatifs exprimant le mépris associé au ridicule ${ }^{13}$. Ce pourcentage indique donc un possible effondrement de l'ethos du présidentiable, tout au moins parmi cette tranche de la population.

D'ailleurs, les plus favorisés (le plus de likes) font usage d'ironie. Ainsi le tweet ci-dessous mentionnant, en implicite que Fillon ment au sujet du suicide :

(1) Petite précision : Aucun média n'a annoncé le suicide de Pénélope Fillon. (27 replies, 171 retweets, 198 likes)

Cependant, le tweet le plus partagé et le plus aimé demande si le suicide de Penelope Fillon a vraiment eu lieu, de manière apparemment neutre ( $c f$. notre section 2.3.):

(2) Fillon au 20h : «On a annoncé le suicide de ma femme mercredi matin sur les chaînes de télévision » C'est vrai ? (330 replies, 7853 retweets, 5619 likes)

Les tweets partagés se limitent à circuler des liens ou d'autres informations, le plus souvent encore avec ironie ou humour, la plateforme s'avérant d'abord être une source nouvelle d'informations plutôt que de débat :

(3) Une rumeur de suicide de Pénélope Fillon évoquée sur des chaines TV ? Fillon en mode « fait alternatif » à la Trump. (71 replies, 849 retweets, 479 likes)

Les quelques tweets qui prennent la défense de Fillon sont peu partagés et peu aimés ; ainsi la réponse de M. de Jessey, porte-parole de Sens commun, qui rejette la faute sur les médias, est retweetée 4 fois avec 1 like :

\footnotetext{
${ }^{13}$ Les autres tweets en défense de Fillon sont aussi sur le mode négatif.
} 
(4) « suicide» Pénélope (...) Mediapart qui annonce que Pénélope Fillon est en garde à vue. C'est le journalisme wishful thinking !

L'ironie principalement atteste d'une vague anti-Fillon sur Twitter, et cet antifillonisme est confirmé par l'analyse des fréquences et des spécificités comme le propose la section ci-dessous.

\subsection{LA SCÉNOGRAPHIE VERBALE DES TWEETS}

Afin de cerner dans son ensemble le cadre discursif des tweets, nous avons procédé à une lecture à l'aide du logiciel Termostat. Celui-ci nous permet de visionner le critère de fréquence et celui de spécificité. La fréquence traditionnellement liste le vocabulaire typique du genre de média, ici c'est la fonction de partage (retweets, "partagé ») et d'évaluation (like) qui définissent Twitter. Le vocabulaire spécifique aux tweets sur Fillon est donné dans le tableau 1 et il a été classé selon trois thèmes $^{14}$ :

- Trump français : la personnalité de l'homme politique ;

- Évaluation ironique : destruction de l'ethos de l'homme politique ;

- Fausses nouvelles : caractéristiques de la campagne.

Tableau $1:$ Les thèmes dominants dans le corpus de tweets

\begin{tabular}{|c|c|}
\hline Thème & Expressions linguistiques (en grasses les plus fréquentes par catégories) \\
\hline Trump français & Trump (108) \\
\hline $\begin{array}{l}\text { Évaluation négative } \\
\text { (autre que Trump) }\end{array}$ & $\begin{array}{l}\text { Conscience morale (17); suicide politique (19); délit d'intox (6). Affabula- } \\
\text { teur (2); mytho (22) ou mythomane (4) }\end{array}$ \\
\hline Fausse nouvelle & $\begin{array}{l}\text { Adjectifs : fictif (45) : d'imaginaire (27) ou d'annoncé (12). } \\
\text { Noms : rumeur (86); intox (60), hoax (3); fait alternatif }(14) \text {, fake news }(75) \text {. } \\
\text { Motivation possible de la rumeur : le calendrier judiciaire (28), un jeu sordide } \\
\text { (10) ou lames de rasoir (18). } \\
\text { Types de fausses nouvelles : le nombre de manifestants exagéré lors de son } \\
\text { discours (18), les autres erreurs de Fillon (38), je ne suis pas autiste (38), les } \\
\text { emplois fictifs (15); l'affaire des costumes (12). }\end{array}$ \\
\hline
\end{tabular}

Selon ce tableau, la thématique se limite à jouer sur le nom propre Trump, à l'évaluation négative de François Fillon (autre que la comparaison avec Trump) et à la circulation de toutes les fausses nouvelles que lui-même a fait circuler, ces trois thèmes contribuant à la destruction de l'ethos de l'homme politique.

${ }^{14} \mathrm{Cf}$. tableau 1. Il est à noter que les emplois fictifs de Madame Fillon ne prennent pas autant d'ampleur que les fausses nouvelles. 
La comparaison de l'homme politique français avec le président américain domine nettement ${ }^{15}$, et permet nombre de jeux de mots comme l'attestent les tweets rassemblés ci-dessous :

(5) Fillon fait du Trump, une réalité alternative digne de \#Trump, \#Trump et \#Fillon doivent avoir le même forfait, Méthode Trump, François \#Fillon fait du \#Trump, Fillon en mode « fait alternatif» à la Trump ou bien Fillon notre Trump «à la française $»$.

Cette analogie a été l'origine de nombreux néologismes (se trumper, trumpisation, trumperies, trumpillon). Si la présence de ces nombreux jeux de mots marque le caractère ludique de Twitter, cette métonymie a une axiologie hautement négative puisque Trump est un homme politique décrit dans la presse comme un impulsif, le contraire de la stabilité, une qualité exigée de l'électorat français selon le Bart (2009).

La récurrence d'une telle métonymie permet la cristallisation de l'image de Fillon en un raccourci ravageur comme l'indique le thème 2 : Évaluation de François Fillon. L'homme politique français devient un « mytho » (6-9), marquant l'incompatibilité de la personne avec les responsabilités d'un président de la république :

(6) En + d'être magouilleur. Parano et mytho, François est encore en train de mythoner, mytho un jour mytho toujours, etc. ;

(7) la Mythomanie le seul pays que \#Fillon pourrait diriger ;

(8) Fillon en mode mytho du début à la fin ;

(9) C'est un gros mytho \#Fillon ils étaient 200 000, les chaînes TV parlaient du suicide imaginaire de Pénélope et là encore un jeu rêvé par lui ${ }^{16}$.

Le tableau 1 nous signale aussi l'effet boule de neige d'une rumeur: la fausse nouvelle du suicide de sa femme a fait émerger de nombreuses autres fausses informations. Ainsi, si le chiffre exagéré de 200000 personnes pour la manifestation de soutien au Trocadéro à son égard ${ }^{17}$ n'était peut-être pas passé inaperçu, l'évocation d'un jeu dans le Vaucluse (Lancer de lames de rasoir) qui consistait à envoyer « des lames de rasoir au lance-pierres dans les jambes des filles $»^{18}$, jeu qui semble inexistant, avait été peu relayée dans les médias; il se trouve déplacé ainsi au centre de la tempête médiatique. Cette diversité des mensonges achève la caricature de l'homme politique probe en parfait « Pinocchio ».

\footnotetext{
${ }^{15}$ Le président américain et son entourage sont connus pour l'expression « faits alternatifs » (de la part de sa porte-parole) et pour fabriquer des nouvelles ou lancer des rumeurs.

${ }^{16}$ Fillon est aussi accusé de menteur, mais cette accusation semble moins dommageable à l'homme politique car le menteur sait ce qu'il fait alors que le mytho est malade.

${ }^{17}$ « Faut dire 200000 ». Et soudain, ils furent 200000 sur une place pouvant contenir 45000 personnes. \#Trocadéro @HugoClement \#Quotidien pic.twitter.com/JnFbk4Jb4B.

${ }^{18} \mathrm{http}$ //www.francetvinfo.fr/politique/francois-fillon/affaires-fillon/suicide-de-penelope-annonce-ala-television-nombre-de-manifestants-calendrier-judiciaire-trois-intox-de-francois-fillon_2083481.html
} 
Notre rapide lecture du cadre discursif décrit par les tweets indique donc une dominance des discours contre l'homme politique. Si nous avons pu en quelque sorte tester le pouls de Twitter au mois de mars 2017, en revanche, étant donné les peu de caractères possibles et l'uni-polarité des messages, nous n'avons pas pu établir l'argumentation travaillée par les militants des Républicains pour restaurer l'ethos de leur champion.

Notre analyse dans la section suivante compense cette absence en reprenant les commentaires des articles les plus partagés dans les tweets. Ce deuxième corpus nous permet de cerner les stratégies argumentatives des supporters de Fillon.

\section{STRATÉGIES ARGUMENTATIVES SUR LES FORUMS}

Dans son étude portant sur le retravail d'ethos de Ségolène Royal lors de sa défaite aux élections en 2007, Amossy $(2014$, p. 26) avait identifié trois stratégies de réparation de l'ethos, notamment:

- le déni ou la fuite de la responsabilité ;

- la réduction de l'offense ;

- l'attaque contre l'accusateur.

Notre deuxième corpus attestera la présence de celles-ci, tout en les complétant.

\subsection{CORPUS DES COMMENTAIRES ET GÉNÉRALITÉS}

Nous avons sélectionné les articles les plus partagés dans les tweets étudiés plus haut et rassemblé les commentaires postés comme réactions à ces articles, tous relatifs à l'affaire Fillon.

Tableau 2 : Articles de presse partagés et commentés par les internautes

\begin{tabular}{|l|l|}
\hline \multicolumn{1}{|c|}{ Titre de l'article et date } & \multicolumn{1}{c|}{ Lien } \\
\hline $\begin{array}{l}\text { Enquêtes, jeux de mots, faux comptes } \\
(26.01 .2017)\end{array}$ & $\begin{array}{l}\text { http://www.20minutes.fr/high-tech/2002567-20170125-enque } \\
\text { tes-jeux-mots-faux-comptes-affaire-penelope-fillon-dope-crea } \\
\text { tivite-internautes }\end{array}$ \\
\hline $\begin{array}{l}\text { La curieuse théorie qui veut faire } \\
\text { croire que François Fillon a été } \\
\text { «blanchi » (13.04.2017) }\end{array}$ & $\begin{array}{l}\text { http://www.lemonde.fr/les-decodeurs/article/2017/04/13/la-cu } \\
\text { rieuse-theorie-qui-veut-faire-croire-que-francois-fillon-a-ete- } \\
\text { blanchi_5110844_4355770.html }\end{array}$ \\
\hline $\begin{array}{l}\text { Tout comprendre aux affaires Fillon } \\
\text { (12.04.2017) }\end{array}$ & $\begin{array}{l}\text { http://www.lemonde.fr/les-decodeurs/article/2017/04/12/tout- } \\
\text { comprendre-aux-affaires-fillon_5110215_4355770.html }\end{array}$ \\
\hline $\begin{array}{l}\text { Lancer de lames de rasoir, suicide de } \\
\text { Pénélope annoncé à la télévision, } \\
\text { nombre de manifestants }\end{array}$ & $\begin{array}{l}\text { http://www.francetvinfo.fr/politique/francois-fillon/affaires- } \\
\text { fillon/suicide-de-penelope-annonce-a-la-television-nombre-de- } \\
\text { manifestants-calendrier-judiciaire-trois-intox-de-francois-fillon } \\
\text { 2083481.html }\end{array}$ \\
\hline
\end{tabular}


Le corpus compte environ 57450 mots. Un dépouillement manuel pour identifier les stratégies indique une plus grande diversité dans les prises de position des commentateurs que dans notre corpus de tweets. Les positionnements pro Fillon, que nous privilégions dans cette section, reprennent ou devancent les grandes lignes de défenses de l'homme politique. Les commentaires ont pour but soit de retravailler l'ethos de Fillon, soit de détruire l'ethos des opposants de Fillon. Pour le premier objectif, trois stratégies, par ordre décroissant, sont présentes pour retrouver la crédibilité et la légitimité :

- victimiser Fillon (évoquer l'empathie envers Fillon);

- minimiser la faute (évoquer la faiblesse humaine, donc la sympathie pour Fillon);

- redéfinir les accusations (la dignité et l'honnêteté de Fillon sont intactes).

D'autres défenseurs de l'homme politique choisissent d'attaquer l'ethos des opposants de Fillon, que ce soit les commentateurs ou les opposants politiques de Fillon. De fait, les attaques contre les accusateurs étaient parmi les stratégies de la femme politique, et nous précisons ici les moyens mis en place pour les construire :

- accusations ad hominem pour arrêter les commentaires par l'humiliation du commentateur ;

- accusations contre l'opposant politique soutenu par le commentateur ;

- accusations de favoriser les extrêmes en attaquant Fillon et ainsi Fillon serait le moindre mal.

Il va sans dire que les différentes argumentations s'entrecroisent dans les messages : on allie à la fois la victimisation et les accusations contre les autres hommes politiques par exemple.

\subsection{STRATÉGIE DE VICTIMISATION : EMPATHIE POUR FILLON, VAILLANCE DES MILITANTS}

La stratégie de victimisation alliée à celle du complot est la plus répandue dans nos données, stratégie aussi employée par Fillon qui a d'abord évoqué l'amour pour sa femme, puis le suicide d'un autre homme politique (Bérégovoy) comme stratégie de défense. De cette manière, les commentateurs disculpent Fillon de ses responsabilités puisqu'il est avant tout la victime d'une campagne politique orchestrée contre lui. Cette campagne serait organisée notamment par les médias qui seraient en faveur de E. Macron. En (10), le déni des accusations est clairement exprimé, faisant appel au bon sens et annonçant aussi l'argument majeur avancé pour sauver la présidentiabilité de Fillon, que ces fausses accusations sont orchestrées par les opposants :

(10) Mentez ! Mentez ! Il en restera toujours quelque chose. Il est évident que Fillon dérange partout, et c'est pourquoi il sera élu. Les gens ne sont plus des Bœufs, que l'on mène sagement à l'abattoir. 
En (11), les sympathisants mettent en exergue la résistance et l'ethos d'un homme politique courageux qui reste encore debout malgré le bashing injuste et orienté politiquement de la part des médias.

(11) malgré tous vos efforts, FF est encore debout, mais le «bashing » continue, et pendant ce temps là, on évite de parler des vrais problèmes.

En (12), les rumeurs seraient une distraction pour éviter que l'on parle des problèmes d'autres candidats, notamment ceux de E. Macron :

(12) (les vrais problèmes) de ceux des autres candidats, ou plus exactement de l'autre, l'histrion christique à l'arche de Noé.

En (13), les commentateurs avancent l'argument de la partialité politique des médias qui soutiendraient les socialistes par l'intermédiaire du candidat Macron. La presse et les médias sont donc accusés de faire une campagne politique contre Fillon explicitement :

(13) Il est vrai que pendant que l'on parle de cela on tait les problèmes d'E. Macron (bien que Ministre des Finances, incapable de remplir correctement son ISF - et comme par hasard à son avantage !) et du fait que sa candidature est téléguidée depuis le début par F. Hollande... mais chut il ne faut pas le dire, ce serait politiquement incorrect. Vous tombez dans le piège des médias, vous ne voyez pas qu'ils veulent que vous votez Macron, c'est leur but faire passer Macron !

Pour renforcer leurs arguments complotistes, les internautes semblent associer des faits similaires, des coïncidences de révélations de scandales contre les grands favoris des élections dans le passé ainsi en (14) ou font des comparaisons entre candidats pour la même élection et le traitement médiatique différent (15). De manière constante est avancé l'argument des médias à la solde du candidat Macron :

(14) C'est troublant non en 2012 DSK et 2017 Fillon on peut se poser des questions quand même !!!!

(15) Macron est à l'ordre du jour uniquement dans le sens positif. Vous voyez bien qu'il a le vent en poupe. Fillon est l'homme à abattre. Les autres font de la figuration.

Cette dénonciation de complot permet aux internautes d'affirmer leur résistance à la manipulation (16) et de continuer à voter pour " leur » candidat qui incarne toujours les vraies valeurs du parti par son programme : on ne vote pas pour un homme, mais un programme (17). Cette posture de vaillance face aux rumeurs et de droiture dans le choix politique coûte que coûte est la plus positive trouvée dans le corpus :

(16) Moi aussi, je voterai pour qui je veux et personne ne me poussera à voter pour un tel ou un tel, ce que les Français devraient savoir ne pas se laisser influencer par personne. 
(17) Je suis assez surpris de voir que des articles a charge essentiellement sur fillon 1, le pen,,tres peu sur leur programme a l'approche du scrutin.j'ai un sentiment fort desagreable sur votre impartialite.chaine publique a la solde de qui!!!!

L'acharnement supposé des médias sur les candidats est donc possiblement une stratégie de manipulation des choix politiques : Fillon est une victime d'une campagne médiatique et politique savamment orchestrée par E. Macron ; les partisans ne failliront pas, ne tomberont pas dans le piège des médias et ne changeront pas leur vote.

\subsection{DÉNI OU MINIMISATION DE LA FAUTE : SYMPATHIE POUR FILLON}

La deuxième stratégie consiste à nier la faute ou à la minimiser afin de sauver le branding d'un homme honnête dont a bénéficié Fillon avant les rumeurs ainsi dans le commentaire (18) où les internautes, pour atténuer les conséquences ou bien pour « dédouaner» complètement Fillon, avancent que « ce qu'il a fait était légal et permis par la loi »:

(18) À présent, Fillon attire encore plus la sympathie, le reproche sur ce qu'il avait dit sur le retrait d'un candidat suite à une mise en examen est instrumentalisé et apparaît de plus en plus injustifié, CE QUI COMPTE, C'EST QU'IL N'A RIEN FAIT D'ILLEGAL et N'EST DONC PAS CONCERNE.

La compétence (argument de présidentiable) est mise en avant afin de répondre aux rumeurs qui attaquent la crédibilité, autre qualité du présidentiable :

(19) N'en déplaise aux moralisateurs, nous votons pour un programme et un homme capable de s'entourer pour l'appliquer, et ce n'est pas une histoire de costume, même chers, ou le fait d'avoir embauché sa femme ce qui n'est pas illégal, qui changera quoi que ce soit pour ce qui me concerne.

Le déni de la faute consiste aussi 1) soit à déformer les faits, en avançant comme argument que la réserve parlementaire est une somme dont les élus ont droit de dépenser selon leur bon vouloir (20), 2) soit à impliquer que celle-ci est pardonnée (21) puisqu'elle a été avouée, ce qui mène donc à l'absolution du candidat :

(20) Détournement de fonds publiques c'est un peu exagéré ?? C'est de l'argent que l'on DONNE aux parlementaires et ils peuvent l'utiliser comme bon leur semble donc? Qu'auriez vous fait à sa place ?? Il n'a pas piqué dans la « caisse».

(21) Fillon a fait une erreur, il l'a reconnu ! pourquoi ne pas lui accorder sa chance alors que la justice l'accorde à des voyous, des assassins, des terroristes ?

La stratégie de minimisation inclut la comparaison avec de véritables crimes ( $i l$ n'a pas tué, il n'a pas piqué dans la caisse) en (20,22), ou la banalisation de celle-ci (23) à travers la naturalisation du mensonge dans la vie : 
(22) Il n'a tué personne...

(23) Il y a mensonges, et mensonges, quelquefois, on n'a pas le choix.

Les affaires qui concernent Fillon deviennent des anecdotes qui relèvent du sensationnel (24) et ne devraient pas faire l'objet d'articles dans des journaux sérieux :

(24) Nous sommes la risée du monde dont les medias sont les pourvoyeurs en se focalisant que sur le sensationnel qui fait vendre. pouvez-vous vous focaliser sur les programmes, c'est a l'heure actuelle la seule chose qui nous interresse.

Même si la faute est réelle, même si elle est grave et ne constitue pas seulement un mensonge de vie courante, le dernier argument est celui de la mise en contexte politique : les autres politiques « font exactement la même chose » (25), la preuve en est les chiffres célèbres du coût du coiffeur de F. Hollande, le président précédent :

(25) Quand on pense que tous les parlementaires qu'ils soient de gauche, de droite, du FN et j'en passe font exactement la même chose sinon pire ça devient ecoeurant. M. Hollande avec son coiffeur à 9000 euros par mois et avec notre argent personne ne dit rien quand on fait le compte c'est une belle somme 540000 euros.

Enfin, ces commentaires permettent de répondre par médias interposés aux tweets vus dans la section précédente de ce chapitre, tweets accusant l'homme politique d'être mythomane. À la caricature malveillante de Fillon se substitue l'incrédibilité et l'irresponsabilité des médias, dans la tentative de reconstruire une image du candidat conforme au profil d'un présidentiable (Amossy, 2014, p. 27) en (26) :

(26) Mais comme on veut encore nous le faire croire c'est Mr Fillon le Mythomane et nos bons médias les saints !!!!

Ces tentatives, soit de déni ou de minimisation de la faute pour re-crédibiliser le candidat, soit d'orientation de la présidentiabilité vers l'argument de compétence sont souvent accompagnées d'attaques en règle contre les opposants, comme nous avons pu le constater dans les citations précédentes et qui font l'objet de la section suivante.

\subsection{ATTAQUES CONTRE LES OPPOSANTS : L'HUMILIATION}

Déjà en (3.2.) il a été permis de constater que la stratégie de victimisation dénonçant un complot s'accompagnait d'attaques en touche contre Macron, le soidisant protégé des médias et supposément le candidat des autres commentateurs. Celui-ci devient la cible des commentaires caustiques qui ne manquent de l'identifier à F. Hollande : la malhonnêteté de Fillon devient celle de Macron, qui de plus est sans personnalité (le clone de Hollande) et donc incapable de diriger le pays : 
(27) Oh arrêtez ça suffit ! Lâchez lui les baskets et occupez-vous de Macron ! Il ne ment pas, il est si honnête, il est capable de diriger notre pays le clone de Hollande ?

Les attaques peuvent aussi concerner le commentateur lui-même, ainsi en (28) où il est impliqué que l'interlocuteur « roule » pour un autre candidat et fait donc des accusations gratuites par objectif politique de décrédibilisation :

(28) Le palmipède est un journal de gauche et qui ne s'en cache pas, accessoirement satirique, mais tout est relatif, question subsidiaire, quel est votre candidat ?

Culpabiliser l'autre intervenant par des sous-entendus et pour des fautes présumées permet aussi de trivialiser les fautes réelles de Fillon :

(29) Une étourderie ça arrive... T’as jamais oublié de déclarer un truc Gilbert ???

De vastes généralisations permettent d'accuser tous les opposants et les médias de décontextualiser les dires et de toujours déformer les paroles ; les autres commentateurs deviennent des «types » qui colportent eux-mêmes des fausses nouvelles :

(30) Reprenez la vidéo de l'émission, ce n'est pas cela qu'il a dit mais « on suppose que ... » ce qui n'est pas la même chose. Pourquoi toujours déformer la vérité. Voilà en quoi les réseaux sociaux sont un système pourri et pervers car il y a toujours un type qui annonce «je tiens cela d'un ami qui connaît le cousin d'un ministre qui... » C'est grave.

L'hostilité perçue dans ces commentaires envers l'autre et l'humiliation éventuellement ressentie par les récepteurs sont des tactiques connues pour faire taire l'opposition. La dernière stratégie dans la section suivante consiste de même à faire taire l'opposant mais par le biais de la peur raisonnée et politique.

\subsection{LA CONTRE PRODUCTIVITÉ DES RUMEURS : LA PEUR}

Brandir l'argument d'un deuxième tour confrontant Marine Le Pen à Jean-Luc Mélenchon, les deux personnes politiques décrites dans les médias comme représentant les extrêmes, permet de faire naître l'angoisse de trouver deux extrêmes au $2^{\mathrm{e}}$ tour :

(31) $\mathrm{Vu}$ que personne ne veut de votre Emmanuel Hollande, tout ce que vous allez réussir à faire avec votre propagande mensongère à 2 bitcoins, c'est nous amener à un deuxième tour Mélanchon-Le Pen, les deux extrémistes et anti-européens de surcroit. C'est du propre!

Cette peur est aussi mise en avant dans le cas où Macron gagne. L'élection de Macron s'associe à cinq ans d'enfer pour le pays : un enfer social avec des individus 
dangereux laissés en liberté ; un enfer économique puisque le désastre des socialistes va être perpétué. Ce discours fortement polarisant vise à culpabiliser les électeurs potentiels de Macron :

(32) Un multi récidiviste fichier $\mathbf{S}$ radicalisé, ultra dangereux qui avait déjà tenté d'assassiner un policier. (...) Merci F. Hollande. À part se précipiter pour annoncer un hommage national qu'a t'il proposé, que n'a t'il fait ? Voter Macron ce sera remercier les socialistes pour ces 5 années d'imposture. Voter Macron ce sera valider cette idéologie socialiste laxistes. (...) Voter Macron se sera valider son implication dans ce gouvernement ( 3 ans en tant que conseillé, 1 an en tant que son ministre de l'Économie).

Pour résumer notre propos, les topoï argumentatifs discutés ci-dessus dans notre corpus sont identiques à ceux identifiés par Amossy (2014), notamment :

- le topos de déni fondé sur la victimisation (susciter l'empathie);

- le topos de minimisation de la faute (retrouver la sympathie);

- le topos d'attaque en règle contre les opposants (humiliation).

Nous avons aussi observé l'élaboration d'un scénario catastrophique provoquant l'angoisse du deuxième tour, une stratégie inconnue pour Ségolène Royal, les circonstances étant différentes, puisque l'élection avait déjà été perdue.

\section{UNE MORALE AU PENELOPEGATE?}

L'activité énonciatrice qui a fait l'objet de cette étude illustre les stratégies déployées au fil des tweets et des commentaires, stratégies faisant «varier les rapports entre fond et forme » (Rastier, 2006, p. 112) : un même fait est construit comme une banalité inhérente au système politique ou un acte indigne d'un futur chef d'état. Nous avons aussi conclu que Twitter jouait ici plus le rôle de relais d'informations alors que les forums de journaux offrent un terrain propice pour identifier les stratégies discursives. Dans le contexte de ces derniers, les stratégies identifiées par R. Amossy interviennent dans le but à la fois de susciter diverses émotions pour encourager la clémence envers Fillon et de désamorcer des arguments contre sa présidentiabilité. Nous avons noté, notamment pour essayer de reconstruire sa crédibilité de présidentiable, le déni de la malhonnêteté par une redéfinition de ce qu'est la faute et la minimisation de celle-ci ; de plus, le réajustement de l'image de l'homme politique vers d'autres composants d'une présidentiabilité, la compétence et la légitimité permet aussi d'alléguer le chaos si les autres candidats de par leur inexpérience ou leurs idées arrivaient au pouvoir. L'analyse de nos données reconfirme donc que ce retravail de l'ethos est une recherche équilibriste entre la reconnaissance d'une faute (perte de crédibilité) et la préservation des arguments d'un présidentiable, pour assurer la légitimité et la compétence. Le Penelopegate a aussi cristallisé 
l'importance centrale de la morale pour les électeurs, notion inhérente aussi à la définition originelle d'ethos :

(33) Si l'on veut vraiment changer les choses, il faut avoir un leadership moral et un charisme que Fillon n'a plus (...). Il faut, pour cela, une rigueur morale que Fillon n'a pas. Il dérivera tout autant sinon plus avec un capitaine qui n'a aucune autorité morale pour en diriger l'équipage.

La loi de la moralisation de la politique décidée en juillet 2017 et la fin de la réserve parlementaire votée en septembre 2017 ont été au moins des conséquences reconnues par tous et toutes comme fort heureuses de Penelopegate. 


\section{BIBLIOGRAPHIE}

Adam, J.-M. (1999). Linguistique textuelle. Des genres de discours aux textes. Paris : Nathan.

Amossy, R. (dir.) (1999). Images de soi dans le discours. La construction de l'ethos. Lausanne-Paris : Delachaux et Niestlé.

Amossy, R. (2002). Ethos. In Charaudeau, P. \& Maingueneau, D. (éd.), Dictionnaire d'Analyse du Discours (pp. 238-240). Paris : Seuil.

Amossy, R. (2010). La présentation de soi. Ethos et identité verbale. Paris : Presses Universitaires de France. Retrieved from https://doi.org/10.3917/puf.amoss.2010.01

Amossy, R. (2014). L'ethos et ses doubles contemporains. Perspectives disciplinaires. Langage et société, 149 (3), pp. 13-30. Retrieved from https://doi.org/10.3917/ls.149.0013

Baider, F. (2015). La parole inversée ? Marine Le Pen et son identité-ressource langagière. Nouvelles Perspectives en Sciences Sociales, 11, pp. 217-252. Retrieved from https://doi.org/10.7202/ $1035937 \mathrm{ar}$

Barthes, R. (1962). L'ancienne rhétorique. Communications, 16, pp. 172-223.

Benoit, W. L. (1995). Accounts, Excuses, and Apologies: a Theory of Image Restoration Strategies. Albany : University of New York.

Chauvin-Vileno, A. (2002). Ethos et texte littéraire. Vers une problématique de la voix. Semen, 14 [en ligne]. Retrieved from http://semn.revues.org/2509

Eggs, E. (1999). Ethos aristotélicien, conviction et pragmatique moderne. In R. Amossy (éd.), Images de Soi dans le Discours. La construction de l'ethos (pp. 31-59). Lausanne : Delachaux et Niestlé.

Faye, G. (2017). Affaire Fillon : le tueur s'appelle Macron. Publié sur NovoPress.Info. Retrieved from https://fr.novopress.info/204029/affaire-fillon-le-tueur-sappelle-macron-par- guillaume-faye

Goffman, E. (1973) [1959]. La mise en scène de la vie quotidienne, t. I, La Présentation de soi. Paris : Minuit.

Lebaron, F. (2017). Une affaire d'ethos : le cas Fillon et la crise du néolibéralisme. Mémoire des luttes. Retrieved from http://www.medelu.org/Une-affaire-d-ethos-le-cas-Fillon https://doi.org/10.3917/ sava.039.0006

Le Bart, C. (2009). L'analyse des livres politiques. Questions de communication [en ligne], 15. Retrieved from http://questionsdecommunication.revues.org/646; https://doi.org/10.4000/questionsde communication.646

Lewandowska-Tomaszczyk, B. (2017). Incivility and confrontation in online conflict discourse. Lodz Paper in Pragmatics, 13 (2), pp. 297-312. Retrieved from https://doi.org/10.1515/lpp-2017-0017

Longhi, J. (2016a). Le tweet politique efficace comme mème textuel : du profilage à viralité. Travaux de Linguistique, 73 (2). Retrieved from https://halshs.archives-ouvertes.fr/halshs-01483945/document

Longhi, J. (2016b). Performativité et performance du discours politique de François Fillon. Retrieved from http://www.atlantico.fr/decryptage/performativite-et-performance-discours-politique-francoisfillon-2917207.html

Maingueneau, D. (2004). Le discours littéraire. Paratopie et scène d'énonciation. Paris : Armand Colin.

Perelman, C., Olbrechts-Tyteca, L. (1992) (5 éd.). Traité de l'Argumentation. La nouvelle rhétorique. Bruxelles : Éditions de l'Université de Bruxelles.

Rastier, F. (2006). Formes sémantiques et textualité. Langages, 163, pp. 99-114. Retrieved from http://www. revue-texto.net/Inedits/Rastier/Rastier_Formes-semantiques.html ; https://doi.org/10.3917/lang.163.0099; https://doi.org/10.3406/lgge.2006.2686

Woerther, F. (2007). L'Ethos aristotélicien. Genèse d'une notion rhétorique. Paris : Vrin. 